\title{
LE PROBLÈME DU KALA-AZAR EST-IL RÉSOLU ?
}

\author{
Par Ch. ANDERSON
}

Sous-Directeur de l'Institut Pasteur de Tunis

A lire aujourd'hui certains auteurs, à lire, en particulier, les ouvrages destinés à l'enseignement qui condensent nécessairement les faits, les rapportent en bloc dans des exposés didactiques, tenus qu'ils sont de présenter une moyenne équilibrée pour mieux refléter l'opinion dominante du moment, on pourrait croire, pour ce qui est du Kala-Azar, que son problème, résolu, appartient désormais à l'Histoire. En est-il vraiment ainsi ? N'y a-t-il pas lieu de se méfier d'un écart possible entre les conclusions du laboratoire et les observations de l'épidémiologiste? N'est-ce pas le cas en l'occurrence ?

Déjà, en 1932, nous avions le sentiment que ce que l'on déclarait définitif n'était peut-être qu'un « moment », qu'une phase du problème aux aspects et aux faces multiples. Nous posions alors ainsi la question (1) :

«Le vaste groupe biologique des leishmanioses a dû, lors de sa mise en place sur le plan de nos connaissances, subir l'arbitraire des classifications. C'était une nécessité pour l'étude, mais cette présentation artificielle, classique, des livres, peut masquer l'aspect véritable du problème. Aussi, pour en avoir une image aussi réelle que possible, convient-il de choisir un foyer d'observation. »

Nous suggérions de prendre la leishmaniose viscérale du chien comme base de départ, et nous rappelions que lorsque $\mathrm{Ch}$. Nicolle avait découvert cette affection en 1908, on l'avait, d'enthousiasme, identifiée aussitôt à celle de l'homme, croyant bien tenir enfin la clé de l'énigme.

Ce sentiment était légitime. Les exemples du même ordre en parasitologie étaient déjà nombreux, et l'on voyait ici un nouvel exemple de réservoir de virus, notion qui commençait à faire son chemin.

(1) Algérie médicale, avril 1932.

Ann. de Parasitologie, t. XXVII, No $1-2-3 .-1952$. 
Pourtant, alors que les pourcentages de chiens infestés semblaient confirmer le fait pour certaines régions, trois cas seulement, sur des milliers d'examens, étaient signalés aux Indes, foyer de, KalaAzar par excellence. Parallèlement, toutefois, en réussissant le passage du Kala-Azar infantile au chien, Nicolle, Manceaux et Comte faisaient faire un nouveau pas dans la voie de la première hypothèse. On tenait le virus. Bien mieux encore, on arrivait à le « passer » sur singe, chacal, chat, cobaye, lapin, renard, hamster, mérion, spermophile. Le protocole allait en se simplifiant. En utilisant les animaux réceptifs, tantôt comme donneurs, tantôt comme receveurs, le problème revenait à trouver l'intermédiaire spécifique en faisant varier tantôt le vecteur, tantôt les conditions du transport. C'est alors, au contraire, que tout se compliqua et que, pour l'épidémiologiste, le but parut s'éloigner à mesure que l'on croyait progresser. Certes, des expériences bien menées pouvaient paraître décisives, telles celles de Knowles, de Christophers, Shortt, Parrot, Donatien, Lestoquard, Yung, Patton, Adler qui établissaient le rôle du phlébotome $(P$. argentipes, chinensis, longicuspis, sergenti, taianensis, papatasi, perniciosus, major, macedonicus, tobbi). Mais, à la même époque, de nombreux expérimentateurs obtenaient des résultats troublants avec d'autres vecteurs, rhipicéphales, stomoxes (1), ou par d'autres voies et en variant les animaux sensibles.

On mettait aussi en évidence le rôle possible de la voie digestive (Archibald, Shortt, Anderson), des souillures par le mucus nasal (Forkner), les selles, l'urine. On relatait un cas de transmission héréditaire (Low). En décembre 1932, enfin, dans les Archives de l'Institut Pasteur de Tunis, nous attirions l'attention sur un fait nouveau ressortant d'une série d'observations tunisiennes. En effet, lors de l'interrogatoire (1925) concernant un jeune enfant atteint de splénomégalie et d'anémie, un fait nous avait frappé à propos du régime auquel était soumis l'enfant : l'ingestion exclusive de lait de chèvre. La ponction de la rate ayant montré de nombreuses leishmanies, nous ne pûmes nous défendre, par la suite, de poser systématiquement la question : L'enfant prend-il ou a-t-il pris à certaine période du lait de chèvre ?

Et c'est ainsi que nous eûmes la surprise d'enregistrer que dans 16 observations positives sur 19 et sur un total de 53 ponctions de rate (à la date de décembre 1932), le lait de chèvre figurait seul, alors que dans tous les cas négatifs (34), il n'était pas mentionné par les parents ou tout au moins (2 cas) avec un doute. Sans préjuger du rapport de cause à effet, la relation avec l'ingestion de lait

(1) Anderson. - Expériences non publiées. 
de chèvre semblait exister (1). Si nous ajoutons que ces faits cadraient avec une observation faite depuis longtemps, et sur laquelle Ch. Nicolle n'avait cessé d'attirer l'attention, à savoir la plus grande fréquence des cas dans les races italienne et maltaise (79 sur un total de 98, contre $4 \mathrm{chez}$ les Musulmans et $3 \mathrm{chez}$ les Israélites), et que la chèvre joue un rôle quotidien plus marqué et plus étroit chez les premiers que chez les autres, la tentation était excusable qui nous poussait à prendre cette concordance en considération. On sait d'ailleurs, depuis Dionisi, que le Kala-Azar frappe assez sévèrement les glandes mammaires, et nous avons mis par ailleurs en évidence le rôle joué par la mammite chronique (2) des chèvres dans la transmission d'un autre germe lactophile, le M. melitensis (3). Cette coïncidence, donc, n'a pas cessé de nous intriguer, d'autant que des expériences entreprises aussitôt nous avaient montré que le lait de chèvre pouvait constituer un milieu vecteur favorable pour les leishmanies (4).

De même, des passages de chiens fortement infestés (rate, moelle osseuse +++++ ) à chèvres, par voie intrapéritonéale (5), nous avaient donné trois fois un résultat positif, à l'autopsie, chez de vieux sujets (6). Par contre, toutes nos tentatives sur des bêtes plus jeunes, avant la mise bas en particulier, avaient échoué, les animaux n'ayant pas supporté le choc de l'inoculation (7). Ces résultats nous autorisaient, dans l'ensemble, à conclure que la chèvre est un animal réceptif, dans les conditions expérimentales, et que

(1) Signalons que lors d'une mission en Argentine nous avions pensé au rôle possible du Lama comme réservoir de virus pour $L$. brasiliensis et nous avions prospecté dans ce sens avec le regretté Pr. S. Mazza. Résultats négatifs.

(2) Burnet et Andrrson. - C.R. Ac. des Sciences, t. CLXXVIII, p. 428, 21-1-1924.

(3) Depuis le cas rapporté par MagGione en 1914 d'un cas de K.A. évoluant chez un bébé de 10 mois simultanément avec une fièvre à melitensis, il n'est pas rare que cette association soit rencontrée.

(4) Nous avons tenté aussi le contrôle des troupeaux de chèvres laitières de Tunis, mais sans succès, car nous n'avons pu remettre sur pied, les chevriers se dérobant cette fois, un contrôle analogue à celui que Et. BURNET (1924) avait organisé pour ses enquêtes sur le $M$. melitensis. D'autre part, le milieu NNN que nous utilisions exclusivement alors étant plus sensible aux impuretés microbiennes secondaires qu'au développement leishmanien, les contaminations étaient impossibles à éviter et, le petit nombre de chèvres contrôlées aidant, interdisaient pratiquement toute recherche. La mise en évidence par inoculations purificatrices ne rend que lorsque les cultures sont riches en leishmanies. Or, celles-ci se défendent très mal contre la concurrence microbienne et les inoculations réclament un produit injecté riche en parasites. Aujourd'hui on pourrait s'aider de l'adjonction d'antibiotiques.

(5) 20 cc. d'une suspension en eau physiologique de tissus broyés provenant d'un chien inoculé lui-même, deux mois avant, avec les tissus d'un hamster $(++++)$, à partir d'une ponction de rate $(++++)$ chez un enfant de 2 ans.

(6) Le passage par hamster a-t-il favorisé le virus ? Un rongeur intermédiaire serait-il nécessaire dans le cycle naturel et serait-il le réservoir?

(7) Expériences non publiées. Nous ne devions jamais être à même de reconstituer les conditions d'un semblable protocole expérimental. 
son lait constitue un milieu non défavorable (1), donc vecteur possible.

Désormais, forts de cette hypothèse de travail, nous insistâmes à nouveau près de nos confrères tunisiens pour qu'ils nous adressent toutes leurs observations, en précisant l'alimentation de leurs jeunes malades. Les faits devaient aller en s'accumulant et venir jeter sur le problème du K.-A., en Tunisie, une lumière qui interférait avec celle projetée par les acquisitions expérimentales publiées, parallèlement, un peu partout dans le monde. Nous pouvions répéter dans notre chronique consacrée au sujet dans nos Archives $(2):$ Notre chronique renferme pour nous des enseignements d'ordre scientifique cachés sous l'apparente froideur stérile de la statistique, enseignements sur lesquels nous ne devons pas cesser d'attirer l'attention. »

N'est-il pas troublant de constater que c'est toujours la population italienne (3) qui, en Tunisie, tient toujours le record du plus lourd tribut payé au K.-A. ? Cette notion est une « constante 》; elle vient troubler l'équation du problème qui pourrait cependant paraître résolu depuis la substitution à l'inconnue, d'un invertébré piqueur.

Comment doit-on la rétablir ? Et l'allure démonstrative de la distribution géographique, qui souligne que ce sont dans les seules régions où vit une population d'origine italienne que l'on rencontre du K.-A. (le Nord), alors que le Bouton d'Orient n'intéresse que le Sud et que les phlébotomes sont identiquement distribués partout ? Il faut bien légitimement penser que tout n'a pas été vu et que l'épidémiologiste, qui bien souvent n'est autre ici que le praticien en contact intime et permanent avec les populations, a encore son mot à dire et peut-être le rôle décisif à jouer. De toute façon, ses enquêtes peuvent contribuer à lever l'hypothèque qui grève toujours au moins le côté biologique du problème. Au clinicien, les faits, les chiffres. A l'homme de laboratoire, les cribles qui permettront l'utilisation des matériaux. Certes, on a pu dire que, ne faisant état que de faits tunisiens, nous pouvions être victimes depuis les premières conclusions de Ch. Nicolle d'une « série de coïnciden-

(1) Nous avions eru pouvoir indiquer le lait comme milieu de culture et d'entretien possible. Après plusieurs années, nous devons reconnaître que tel quel, c'est un milieu inégal, d'un emploi déroutant au laboratoire pour un usage régulier.

(2) Archives Institut Pasteur de Tunis, t. XXVIT, p. 96, 1938.

(3) L'hypothèse d'une immunité acquise de longue date dans la Régence chez les Musulmans et les Israélites ne tient pas, car elle ne saurait jouer contre les Italiens seuls. De même nous avons procédé à la recherche des groupes sanguins et nous n'avons pas constaté une sensibilité plus marquée pour tel ou tel groupe comme Artamonov (1929) avait semblé pouvoir le rapporter pour le groupe IV. 
ces ». Il n'en est rien, car, réparties comme elles le sont sur un grand nombre d'années, fruit du labeur d'observations multiples, ces constatations tunisiennes apparaissent indubitablement comme ayant une signification propre, spécifique. La notion particulière qu'elles ne cessent de souligner ne saurait être banale et due au hasard. Elles doivent faire soupçonner, au moins pour la Régence, l'existence d'un facteur qu'il s'agira d'intégrer un jour, quand on reconsidérera la question dans son ensemble et dans son aspect universel.

Au cours des années 1938 à 1941, notre opinion devait aller en s'affermissant. Les circonstances nées de l'occupation, puis des suites de la guerre, nous avaient cependant mis dans l'impossibilité de garder le contact avec les praticiens, eux-mêmes pris dans le remous, lorsque, en 1950, nous eûmes connaissance du rapport de P. Giraud, J. Ranque et $H$. Cabassu (1), sur l'épidémiologie de la leishmaniose viscérale humaine méditerranéenne. Ces auteurs commencent ainsi, en bref, leur exposé :

" Si la thérapeutique de la leishmaniose viscérale humaine est entrée dans la voie des réalisations presque parfaites, par contre, les modalités de la transmission de la maladie sont encore loin d'être parfaitement connues. Il convient donc de serrer le problème d'aussi près que possible par l'étude de documents précis fournis par l'observation clinique et l'expérimentation. A ce titre, l'étude du foyer marseillais nous paraît être un fait de premier ordre, en raison du nombre de cas observés et de la durée de ces recherches, échelonnées sur 27 ans. »

Eux aussi relatent la prédominance des cas de un à trois ans et croient pouvoir dire : ...Ces résultats ne sont pas très en faveur de l'action d'un insecte ailé comme le phlébotome qui pique aussi bien et même plus facilement les enfants de moins d'un an que ceux un peu plus âgés. Ils notent également la prédominance des cas en banlieue et à la campagne. Ils s'étonnent aussi du fait que des régions comme celles de Toulouse, de Bordeaux et du Sud-Ouest paraissent épargnées. Ils insistent tout particulièrement sur le fait que la maladie leishmanienne coüncide géographiquement avec la diffusion de la mélitococcie. Et ils concluent en disant qu'ils ne considèrent pas la question de l'hôte intermédiaire, vecteur du $K .-A$. méditerranéen, comme définitivement résolue.

On ne peut pas ne pas être frappé de la similitude de nos conclusions de 1938 avec celles des auteurs marseillais (1950), qui eux

(1) Rev. de Path. comp., avril 1950. 
aussi ont pris comme base de référence la leishmaniose viscérale du chien. En dehors de cette identité de vues que nous soinmes heureux de relever, car la valeur de nos constatations s'en trouve exaltée, nous enregistrons aussi qu'elles sont formulées dans des conditions homologues, à 12 ans de distance, et par des auteurs qui ont étudié patiemment, comme nous, pendant de longues années, un même et vaste foyer.

Il semble donc bien que la question n'ait pas progressé. Nos collègues marseillais ont cru devoir faire appel, eux aussi, au concours des cliniciens, et ce que nous écrivions en 1938 reste donc d'actualité :

«Une fois de plus, nous appelons à l'aide nos confrères praticiens (1). Par leurs fonctions, ils touchent de plus près le "mystère" et le laboratoire, avec ses méthodes, peut n'être ici que le prolongement de leurs observations. Nous insistons d'autant plus que nous pouvons craindre que des observations de K.-A. nous échappent. Or, toutes ont leur valeur. Qu'on veuille donc bien nous les adresser toutes, en se pénétrant de cette vérité élémentaire qu'en matière de prospection, le plus petit détail, négligeable en apparence, a son importance, dût-il ne la tirer avec éclat que lors de la confrontation générale finale. Puissions-nous, dans un jour proche, avoir à célébrer une nouvelle victoire de cette alliance renouvelée de la clinique et du laboratoire. »

Nous devions d'ailleurs, quant à nous, revenir à la tâche expérimentale. Successivement, nous reprîmes nos essais de transmission par contact, par contage, par ingestion, puis par l'intermédiaire de la tique, des stomoxes (2).

Pour des raisons indépendantes de notre volonté, nous ne pouvons pas entrer dans le détail des protocoles, inédits.

Nous pouvons toutefois dire que le pourcentage des résultats positifs l'emporte de beaucoup sur les échecs, ceux-ci étant imputables en majeure partie soit à des morts prématurées chez les animaux d'expériences, soit aux conditions par trop précaires de certains essais que nous voulions réaliser empiriquement dans des conditions aussi voisines que possible de celles rencontrées dans la nature. Quoi qu'il en soit, au terme de notre exposé, nous gardons,

(1) On peut adresser le même appel aujourd'hui aux confrères de Madagascar et à ceux d'Iran, comme nous avons déjà alerté nos confrères Siciliens au cours d'une mission à Palerme (1948).

(2) Stomoxes issus d'élevages aseptiques selon la technique de Worman (Arch. I. P. T., t. XVI, p. 347). Nous eûmes deux hamsters + (St. calcitrans et Lyperosia irritans). Nous attirons l'attention des chercheurs sur l'intérêt que présente la technique du regretté Wolman en dehors de son application au K.A. Des expériences avec différents hétéroptères de Tunisie restèrent négatives. 
quant à nous, cette impression qu'il n'est pas impossible que, dans la transmission, le contact direct, la tique, les stomoxes, la voie digestive puissent jouer leur rôle, en Tunisie tout au moins, sans rejeter pour autant la part du phlébotome (1), et nous continuons à nous croire fondé à ranger pro parte le lait de chèvre (2) parmi la liste des suspects, tant que des expériences aussi décisives que celles que nous avons poursuivies pour éliminer la puce et la punaise ne nous auront pas contraint à y renoncer. Et, ainsi, nous reposons le problème tout entier dans son vaste cadre biologique, cadre dont nous ne pouvons pas encore ou ne savons pas encore prendre une vue panoramique (réservoir, intermédiaire, vecteur) (3) et que nous continuons, de bonne foi, à occulter par des expériences en ordre dispersé, dont le caractère parfois séduisant, mais qui reste fragmentaire, l'homme lui-même ne constituant probablement qu'une impasse, nous incline, à chaque étape, à croire que c'est la dernière. A nous, au contraire, de persévérer.

Si tout n'est pas dit, il nous faut néanmoins conclure et, puisqu'aussi bien c'est notre ancien Maitre que nous honorons aujourd'hui, je ne puis trouver plus émouvante façon de le faire que, d'adapter ici cette exhortation qu'il se plaisait à nous lancer, lors de ses soudaines incursions parmi ses préparateurs :

" Allons, sans cesse, sur le chantier, remettez votre ouvrage... En biologie, surtout, c'est une excellente discipline. »

(1) P. Graud et ses collaborateurs ne prétendent pas plus que nous donner à leurs conclusions une portée universelle et restent, comme nous le disions déjà en 1932, sur le terrain de l'épidémiologiste, réservant le problème de biologie générale entrevu.

(2) Rappelons ici les constatations faites par certains auteurs comme CursoN en Afrique du Sud (B.P., 1927).

(3) Les trois notions restent toujours bien à préciser. 